\title{
A complex additive model of inheritance for Hirschsprung disease is supported by both RET mutations and predisposing RET haplotypes
}

Macarena Ruiz-Ferrer ${ }^{1}$, Raquel M. Fernández, $P h D^{1}$, Guillermo Antiñolo, $M D, P h D^{1}$, Manuel López-Alonso, $M D, P h D^{2}$, Charis Eng, $M D, P h D^{3}$, and Salud Borrego, $M D, P h D^{1}$

\begin{abstract}
Purpose: The RET proto-oncogene is considered to be the major susceptibility gene involved in Hirschsprung disease. Traditional RET germline mutations account for a small subset of Hirschsprung disease patients, but several studies have shown that there is a specific haplotype of RET associated with the sporadic forms of Hirschsprung disease. We have investigated for RET germline mutations and analyzed the RET haplotypic distribution in carriers versus noncarriers of RET germline mutations. Methods: We have screened the coding region of RET in 106 Spanish Hirschsprung disease patients using dHPLC technology. Statistical comparisons of the distribution of RET haplotypes between sporadic patients with and without a RET germline mutation were performed. Results: Nine novel germline mutations and one previously described were identified. A significant over-transmission of the "Hirschsprung disease haplotype" was detected when comparing transmitted versus nontransmitted alleles in the group of Hirschsprung disease triads without mutation. However, no distortion of the transmission of alleles was found in the group of mutated families. Conclusions: These results would be concordant with a complex additive model of inheritance. The whole findings seem to suggest that low-penetrance mutations would be necessary but not sufficient and the additional presence of the "Hirschsprung disease haplotype" could contribute to the manifestation of the disease. Genet Med 2006:8(11):704-710.
\end{abstract}

Key Words: Hirschsprung, haplotype, RET proto-oncogene, germline mutation, dHPLC

Hirschsprung disease (HSCR, OMIM 142623) or aganglionic megacolon, occurring in approximately 1 in 5000 live births, is a neurocristopathy defined by the absence of intramural ganglion cells in the myenteric and submucosal plexuses along variable lengths of the gastrointestinal tract, resulting in functional intestinal obstruction. HSCR most commonly presents in sporadic cases, although it can be familial and may be inherited as autosomal dominant or autosomal recessive, with reduced penetrance, male predominance, and variability of the length of the aganglionic segment. ${ }^{1,2}$ In addition HSCR is frequently associated with many other neurocristopathies and chromosomes abnormalities. ${ }^{2}$

To date, at least 10 genes have been described as associated with sporadic or syndromic forms of HSCR. These "HSCR genes" are generally related to the developmental program of neural crest cells, such as the RET proto-oncogene, glial cell

\footnotetext{
From the ${ }^{1}$ Unidad Clinica de Genética y Reproducción, Hospitales Universitarios Virgen del Rocío, Seville, Spain; ${ }^{2}$ Servicio de Cirugía Infantil, Hospitales Universitarios Virgen del Rocío, Seville, Spain; and ${ }^{3}$ Genomic Medicine Institute, Department of Hematology and Medical Oncology, The Cleveland Clinic Lerner Research Institute, Cleveland, Ohio.

Salud Borrego, MD, PhD, Unidad Clínica de Genética y Reproducción Hospitales Universitarios Virgen del Rocío Avenida Manuel Siurot s/n 41013 Seville, Spain.

Submitted for publication March 13, 2006.

Accepted for publication August 14, 2006.

DOI: 10.1097/01.gim.0000245632.06064.f1
}

line derived neurotrophic factor $(G D N F)$, neurturin $(N T N)$, endothelin 3 (EDN3), endothelin receptor $\beta$ (EDNRB), endothelin converting enzyme 1 (ECE1), transcriptional factors SOX10 and PHOX2B, Smad interacting protein 1 (SIP1) and KIAA1279. ${ }^{2-5}$ However, undoubtedly, the major susceptibility gene for HSCR is the RET proto-oncogene (OMIM 164761), which encodes a receptor tyrosine kinase expressed in tissues and tumors derived from the neural crest and neuroectoderm. ${ }^{2}$ This receptor consists of a 1114 amino acid transmembrane protein presenting an extracellular ligand-binding region, which includes a cadherin-like domain and a cysteine-rich region, and a conserved intracellular tyrosine kinase domain. ${ }^{6}$ It has been shown that RET is the signaling component of a multisubunit complex acting as a receptor for growth factors of the GDNF family, including GDNF, neurturin (NTN), artemin (ARTN), and persephin (PSPN). All these factors activate RET via four different glycosyl phosphatidylinositol-linked GFR $\alpha$ receptors named GFR $\alpha 1$ to 4 and encoded by the GFRA1-4 genes, respectively. ${ }^{7}$

Loss of function germline mutations in RET have been reported to account for up to $50 \%$ of familial HSCR cases and between $3 \%$ and $35 \%$ of sporadic cases. ${ }^{8-13}$ These mutations are distributed throughout different domains of RET and biochemical studies have shown variable consequences such as misfolding, failure to transport the protein to the cell surface, or even a complete abolished biological activity. In summary, 
the results derived from in vitro analyses suggest that haploinsufficiency is the most likely mechanism for the RET mutations associated to HSCR. It is hypothesized that this haploinsufficiency might produce a decrease of all RET-dependent signaling pathways, leading to defects of enteric nervous system development during embryogenesis, resulting in the HSCR phenotype.

Because traditional RET germline mutations accounted for such a small subset of HSCR, we sought to determine if other more common susceptibility factors existed which would predispose to the majority of HSCR. When the variant A45A (exon 2), was found to be highly associated with a large subset of nonfamilial HSCR, ${ }^{14,15}$ it prompted us to hypothesize that this was the associated variant itself or that A45A was in linkage disequilibrium with the putative low penetrance susceptibility locus that would account for the majority of HSCR. Subsequently, we showed that A45A anchors ancestral haplotypes in linkage disequilibrium with such a putative common founding susceptibility locus estimated to be $22-50 \mathrm{~kb}$ upstream. ${ }^{16}$ This was the starting point which led to the identification of a predisposing haplotype located at the $5^{\prime}$ region of RET, whatever the ethnic background, with some functional data suggesting a putative role of the promoter variants $-200 \mathrm{~A}>\mathrm{G}$ and $-196 \mathrm{C}>\mathrm{A} .{ }^{17-22}$

In the present report, we report for the first time a mutational screening in the RET proto-oncogene in a series of HSCR patients from Spain, and with the available data derived from previous studies, ${ }^{19}$ we have analyzed and compared the $R E T$ haplotypic distribution in carriers versus noncarriers of traditional RET germline mutations.

\section{MATERIALS AND METHODS}

\section{Patients and control subjects}

In this study, we have included a total of 106 HSCR patients from Spain (17\% female, $83 \%$ male). Eighty-three of these patients were sporadic cases, while 23 were familial cases belonging to 13 different families. In order to define the exact HSCR phenotype in our patients, we have used the criteria recommended by Chakravarti et al. ${ }^{2}$ Following these criteria, 47 cases were catalogued as short-segment HSCR (52\%), 20 cases as colonic-segment forms (22\%), 14 cases as longsegment (15\%), and 10 cases presented with total colonic aganglionosis (11\%). For subsequent analyses we included the colonic-segment cases into the group of long-segment forms.

In addition, we have also analyzed a group of 100 normal controls comprising unselected, unrelated, race, age, and sexmatched individuals. All of them were healthy voluntary donors, who came to the Hospital for other reasons and did not present any symptom suggestive of HSCR.

An informed consent was obtained from all the participants for clinical and molecular genetic studies. The study conformed to the tenets of the declaration of Helsinki.

\section{PCR and AHPLC analysis}

Genomic DNA was extracted according to standard protocols. ${ }^{23}$ We screened all the RET exons, including intron/ exon boundaries. Primers used for polymerase chain reaction (PCR) amplification and annealing temperatures are summarized in Table 1.

The dHPLC analyses were carried out on an automated HPLC device equipped with a DNA separation column using the WAVE DNA Fragment Analysis system (Transgenomic). PCR products were denatured for $3 \mathrm{~min}$ at $95^{\circ} \mathrm{C}$ and then gradually reannealed by decreasing sample temperature from $95^{\circ} \mathrm{C}$ to $65^{\circ} \mathrm{C}$ over a period of $30 \mathrm{~min}$. Five $\mu \mathrm{L}$ of PCR products were then injected onto the DNASeq cartridge and eluted at a constant flow rate of $0.9 \mathrm{~mL} /$ minute through a linear acetonitrile gradient. DNA fragments were detected by the system's UV detector and analyzed as chromatograms. Oven temperature for optimal heteroduplex separation under partial DNA denaturation was determined for each amplicon using the WAVEMarker software (Table 1).

In addition, those exons with an aberrant wave profile were also screened by dHPLC analysis in a group of 100 normal controls, in order to determine if the variants identified were mutations or just common polymorphisms.

\section{Sequence analysis}

Those samples with aberrant wave profiles were subjected to sequence analysis. PCR products were purified using the Quiaquik gel extraction kit (Quiagen) and sequenced using the DYEnamic $^{\mathrm{TM}}$ ET Dye terminator cycle sequencing kit (Amersham Biosciences) and a MegaBACE ${ }^{\mathrm{TM}}$ automated sequencer (Amersham Bioscience). The alignment of our results with the sequences provided for RET (RefSeq NM_020975.3) was carried out using the software BioEdit Sequence Alignment Editor version 5.0.9.

When a novel RET variant was identified in a proband, all the available family members were sequenced for the appropriate exon in order to define their molecular status regarding such variant.

\section{Statistical analysis}

The distribution of $R E T$ haplotypes comprising the 2 variants within the promoter region $(-200 \mathrm{~A}>\mathrm{G}$ and $-196 \mathrm{C}>\mathrm{A})$ was analyzed and compared between sporadic patients with and without a RET germline mutation. In addition, we searched for a distortion of the transmission of alleles from parents to affected HSCR children, in both mutated and nonmutated groups. The genotyping data were available from previous reports. ${ }^{19}$ Comparisons were performed using the $\chi^{2}$ analysis with Yate's correction, with statistical significance at $P<0.05$.

\section{RESULTS}

We have analyzed the coding region of RET in 106 Spanish HSCR patients using dHPLC technology. A total of 23 aberrant 
Table 1

Amplification and dHPLC conditions for RET genomic sequence analysis

\begin{tabular}{|c|c|c|c|}
\hline Exon/ Primers & $\begin{array}{c}\text { Annealing } \\
\text { Temperature } \\
\left({ }^{\circ} \mathrm{C}\right)\end{array}$ & $\begin{array}{c}\text { dHPLC } \\
\text { Temperature } \\
\left({ }^{\circ} \mathrm{C}\right)\end{array}$ & $\% \mathrm{~B}$ \\
\hline 1F CTAGCCGCAGTCCCTCCA & 62 & 68.6 & 51.1 \\
\hline \multicolumn{4}{|l|}{ 1R ACAGAAAGGCGATTCTGAAC } \\
\hline 2F ССТТАТТСТСАССАТСССТС & 56 & 60.5 & 56.8 \\
\hline 2R AGTGTCAGCGGCTGTGATAA & & 64 & \\
\hline 3F CCCCACAGACCTGACTTCTCT & 67 & 62.8 & 56.6 \\
\hline 3R AAGACCAGCAGTAGCAGGCA & & 63.9 & \\
\hline 4F GCCCCTGTCTGCTTGGTGC & 64 & 65 & 55.8 \\
\hline 4R GGACACTAAACCGACCGAG & & 67.4 & \\
\hline 5F ACTGACCAACGCCCTCTGC & 60 & 63 & 53.9 \\
\hline 5R GCACCTCATTTCCTGGGGG & & 65.3 & \\
\hline 6F ATTGTTGTGCCCCTACCTG & 58 & 64 & 54.3 \\
\hline \multicolumn{4}{|l|}{ 6R CCCCAGACAGGCAATAGGTA } \\
\hline 7F TCTACCCTCAGGCCATTACA & 56 & 61.7 & 58.3 \\
\hline 7R AACCATTTACTGCTGGGTCA & & 63 & \\
\hline 8F TGGTGCTGTTCCCTGTCC & 62 & 65 & 53.5 \\
\hline \multicolumn{4}{|l|}{ 8R CCACCGGTGCCATCGCCCCT } \\
\hline 9F AGCCTGCTGTGTGTCCTGTG & 60 & 62 & 48.2 \\
\hline 9R CCATGCCCTGATTAAACCCT & & 64 & \\
\hline 10F GCGCCCCAGGAGGCTGAGTG & 68 & 64.7 & 50 \\
\hline \multicolumn{4}{|l|}{ 10R GGTGGTGGTCCCGGCCGCC } \\
\hline 11F TGCCAAGCCTCACACCAC & 67 & 64 & 55.1 \\
\hline 11R TCCCTCCCTGGAAGGCAG & & 64.8 & \\
\hline 12F TTCTTCСТCСССТGTCATCCT & 62 & 59.3 & 54.9 \\
\hline 12R TCTTCAGGGTCCCATGCTG & & 65 & \\
\hline 13F GCAGGCCTCTCTGTCTGAACTT & 65 & 63.5 & 54.6 \\
\hline \multicolumn{4}{|l|}{ 13R GGAGAACAGGGCTGTATGGA } \\
\hline 14F AAGACCCAAGCTGCCTGAC & 65 & 62 & 54.6 \\
\hline 14R GCTGGGTGCAGAGCCATAT & & 65.6 & \\
\hline 15F GTCTCACCAGGCCGCTAC & 60 & 62.2 & 54.5 \\
\hline 15R ATGGTGCACCTGGGATCCCT & & 64.4 & \\
\hline 16F AGGGATAGGGCCTGGCCTTC & 66 & 58.6 & 50.3 \\
\hline \multicolumn{4}{|l|}{ 16R TAACCTCCACCCCAAGAGAG } \\
\hline 17F CACTGGTCCTTTCACTCTCT & 59 & 62 & 52.2 \\
\hline 17R GGGAGGGAATGCACACAGAT & & 63.4 & \\
\hline 18F TGTGGTGGGCTGTCCTTCTG & 63 & 61.6 & 52.4 \\
\hline 18R CTGGGGTGAGGCTGGAGTCT & & 62.9 & \\
\hline 19F AGTGACCGGCCATCTCTGT & 64 & 58.1 & 52.2 \\
\hline 19R ATAGTGCAAAGGGGACAGC & & 62.2 & \\
\hline 20F AGTTTTGGTTCTTCAGTGC & 56 & 58 & 53.6 \\
\hline 20R GACTTTCCATTCTCAGCAT & & & \\
\hline 21F GCTTCTTGTCATTCTTCATTGC & 61 & 61 & 53.7 \\
\hline 21R CTTGGCCTCACAAAATGC & & & \\
\hline
\end{tabular}

wave profiles were detected and subsequent direct sequence analysis revealed 13 previously described polymorphisms and 10 different germline mutations.

Among the common polymorphisms detected, 7 were located in coding region, such as A45A (exon 2, rs1800858), V125V (exon 3, rs1800859), A432A (exon 7, rs1800860), G691S (exon 11, rs1799939), L769L (exon 13, rs1800861), S836S (exon 14, rs1800862) and S904S (exon 15, rs1800863); the remaining 6 were localized in intronic regions, such as IVS1 + 53G $>$ A (intron 1, rs12267460), IVS2 + 9G > A (intron 2, rs2435351), IVS4 + 48A $>$ G (intron 4, rs2435352), IVS12 + $47 \mathrm{C}>\mathrm{T}$ (intron 12, rs760466), IVS14-24G $>\mathrm{A}$ (intron 14, rs2472737) and IVS19 + 48A $>$ G (intron 19, rs2075912). All these polymorphisms had been previously evaluated as susceptibility factors for HSCR by cases-controls studies. ${ }^{14,15,24}$

Regarding the RET mutations identified, all of them were novel except for one that had been previously described by $\mathrm{Wu}$ et al. ${ }^{25}$ Both their nature and location varied widely (Figs. 1 and 2 ) and consisted of 2 insertions (both in exon 12), 2 splicing mutations (introns 6 and 13), and 6 nucleotide substitutions which include five missense (exons 3, 4, 6, 13 and 19) and a double change resulting in a silent and a nonsense replacements (exon 10) (Table 2). All the mutations found were shown to be absent in the 100 healthy controls tested. As shown in Table 2, these 10 mutations were detected in 11 independent families, being one of them (A373V, exon 6) present in 2 different pedigrees.

Of the 13 families with familial cases, 3 were mutation positive (Fig. 3), which corresponds to a frequency of $23 \%$. However and surprisingly, in one of these families (HSCR 3), we observed that only one of the two affected members harbored the mutation $Y 791 \mathrm{~N}$, which had been inherited from his unaffected mother and was also present in other apparently healthy family members. Because codon 791 is related to FMTC/MEN $2 \mathrm{~A}$ syndromes, all the carriers of the $\mathrm{Y} 791 \mathrm{~N}$ mutation were examined for clinical and biochemical symptoms of MEN 2 but they were found to be negative.

Among the 83 patients with sporadic HSCR, 8 (10\%) were shown to carry RET germline mutations. Only the 2 insertions detected (740insC and 756insT) were de novo mutations, while in the 6 remaining cases they were inherited from one of their unaffected parents and frequently appeared in other healthy relatives, which is a relatively common feature among complex diseases.

We analyzed a possible correlation between the presence of $R E T$ germline mutations and the length of aganglionic segment in our series. We verified that the frequency of RET mutations was higher among patients with either long-segment or total colonic aganglionosis than in patients with a short-segment form. More specifically, such RET mutations were found in $4 / 47$ short-segment cases (8.5\%), 4/34 long-segment cases $(11.8 \%)$, and in $4 / 10$ patients presenting with total colonic aganglionosis (40\%).

Finally, with the available data from a study previously reported, ${ }^{19}$ we proceeded to analyze the distribution of RET haplotypes, comprising variants at the promoter region of the 

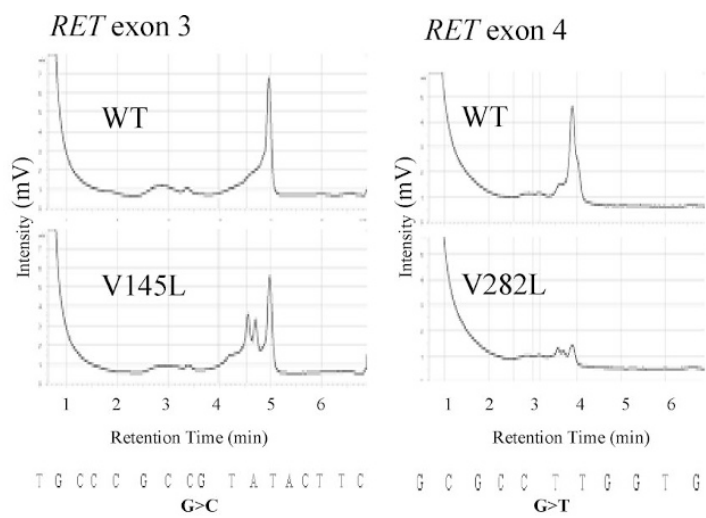

RET exon 10

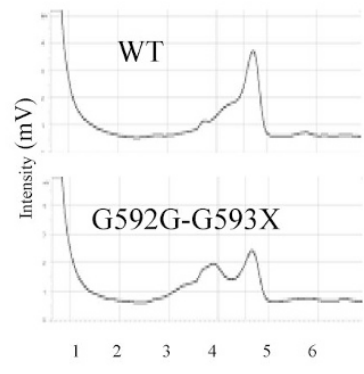

Retention Time (min)

TTG TTG GATIG ACA CG AGCC
RET exon 19

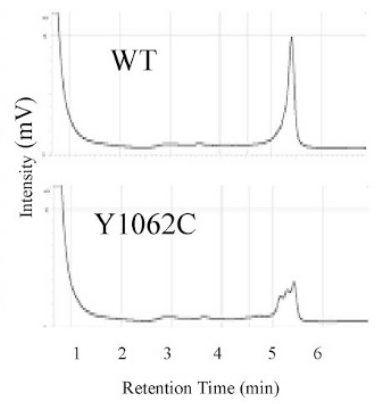

$A A C T C T G T Q Q T A G A$ $\mathbf{A}>\mathbf{G}$
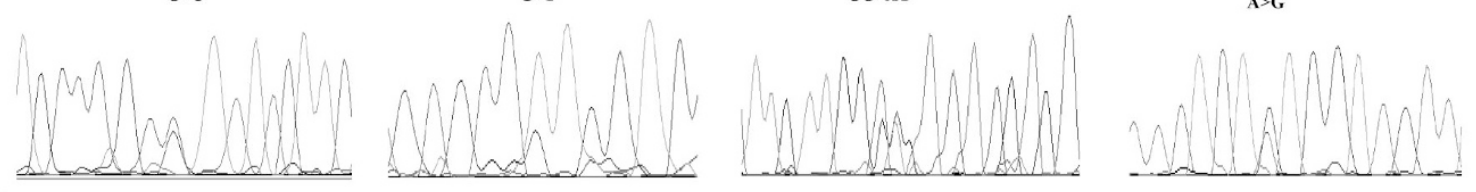

RET exon 6
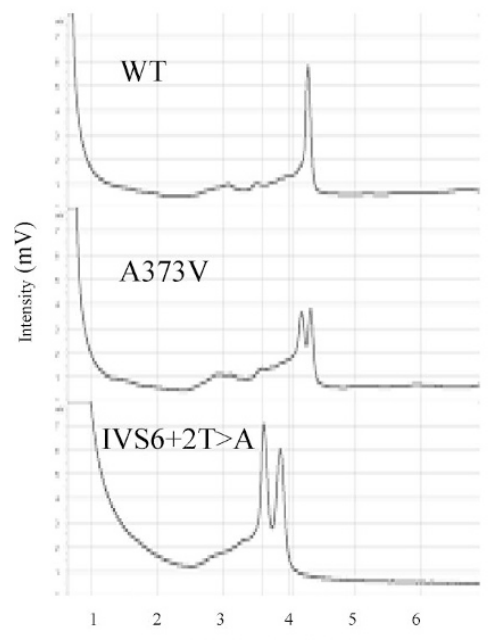

Retention Time (min

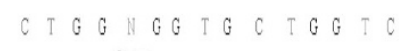

$\mathrm{C}>\mathrm{T}$

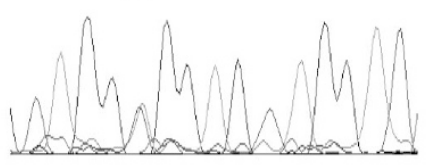

$T G C$ C $\dddot{C} A$ G G

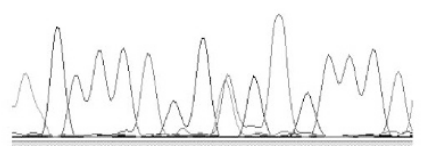

RET exon 12
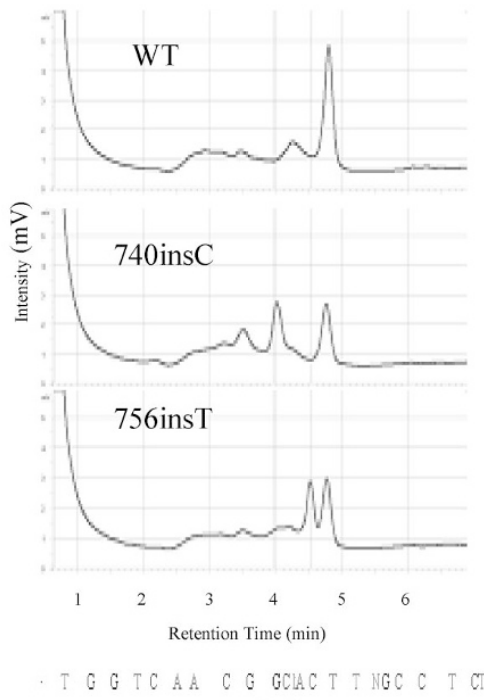

ins $\mathrm{C}$
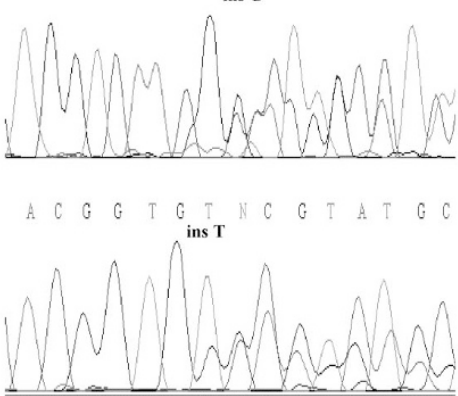

RET exon 13
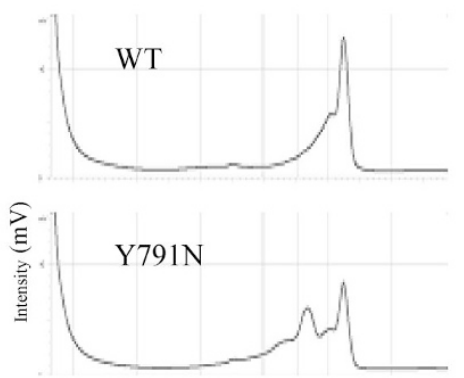

IVS13+19T $>$ C
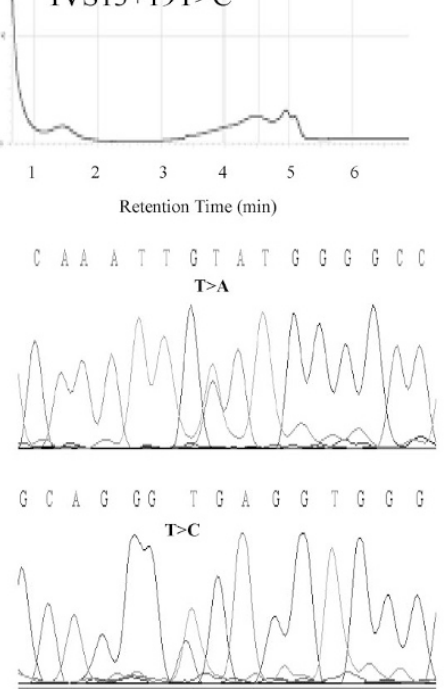

Fig. 1. Denaturating high performance chromatography elution profiles and sequences analysis of the RET mutations in HSCR patients.

gene $(-200 A>G$ and $-196 C>A)$. Based on the presence or absence of RET germline mutations, we divided our series of sporadic patients into two groups (those with traditional germline RET mutations and those without) and made comparisons between them. Although the frequencies of each of the 3 haplotypes found were slightly different in families with traditional RET mutations compared to those without muta- tions (AC: $50 \%$ vs. $62 \%$, GC: $29 \%$ vs. $22 \%$, GA: $21 \%$ vs. $15 \%$ ), such differences did not reach statistical significance $\left(\chi^{2}=\right.$ $0.84, P=0.55)$.

Parental haplotypes were examined in the context of the affected children's haplotypes, so that transmitted and nontransmitted haplotypes were noted and their frequencies compared in each of the groups (Table 3). Similar distortion values 

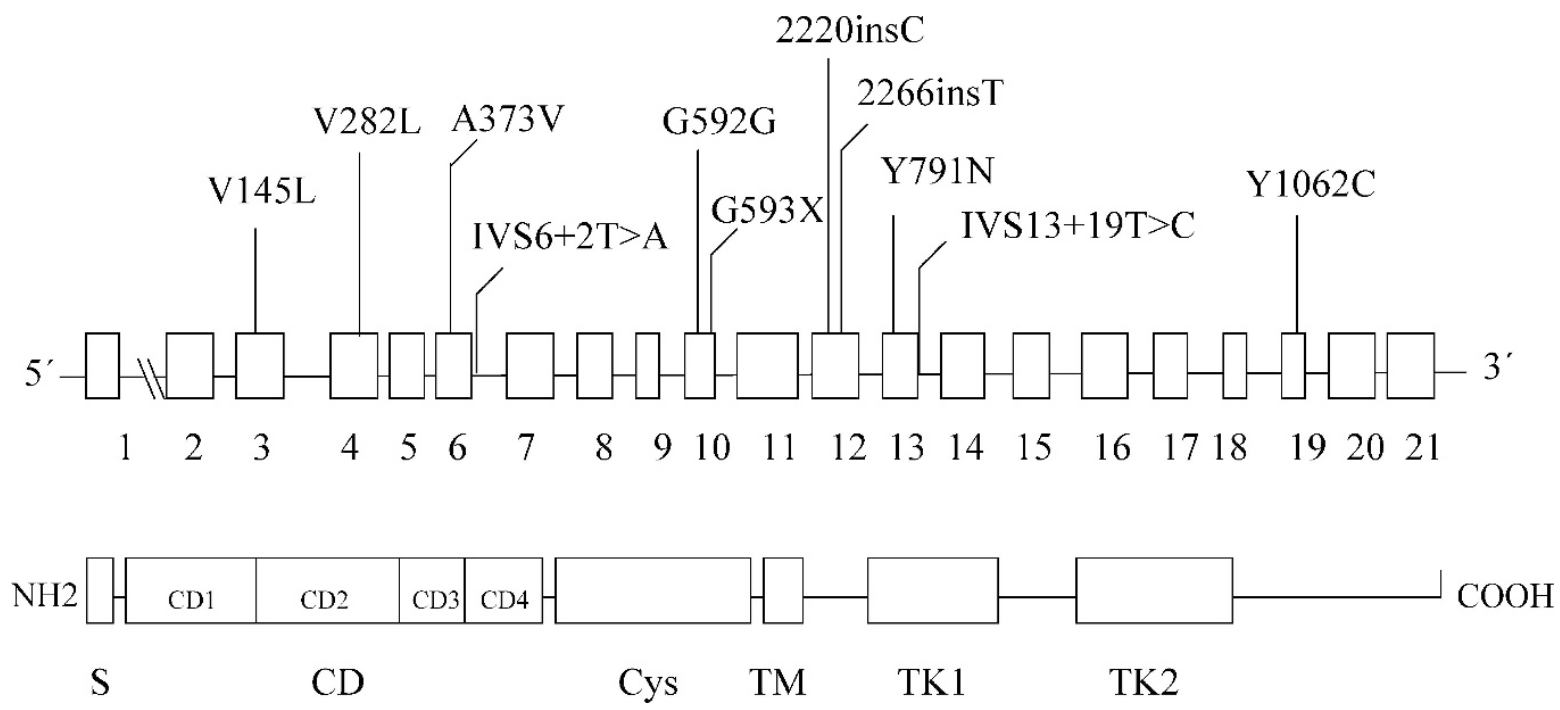

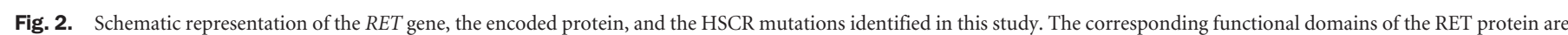
indicated: S, signal peptide; Cd, cadherin-like domain; TM, transmembrane domain; Cys, cysteine-rich domain; TK, tyrosine kinase domain 1 and 2.

Table 2

RET germline mutations identified in HSCR patients

\begin{tabular}{|c|c|c|c|c|c|c|c|c|}
\hline Patient & $\begin{array}{l}\text { Familial/ } \\
\text { sporadic }^{a}\end{array}$ & $\begin{array}{l}\text { Exon/ } \\
\text { intron }\end{array}$ & Nucleotide change & $\begin{array}{l}\text { Amino acid } \\
\text { change }\end{array}$ & $\begin{array}{c}\text { Parent origin } \\
\text { of the } \\
\text { mutation }\end{array}$ & $\begin{array}{l}\text { Presence in other } \\
\text { family members }\end{array}$ & Haplotype & $\begin{array}{c}\text { Length of } \\
\text { aganglionosis }\end{array}$ \\
\hline HSCR 22 & Sporadic & 3 & $c 433 G>C$ & V145L & Mother & No & $\mathrm{AC} / \mathrm{AC}$ & TCA \\
\hline HSCR 128 & Familial & 4 & $\mathrm{c} 844 \mathrm{G}>\mathrm{T}$ & V282L & Mother & No & & Short-segment \\
\hline HSCR 1 & Sporadic & 6 & $\mathrm{c} 1118 \mathrm{C}>\mathrm{T}$ & A373V & Mother & No & $\mathrm{AC} / \mathrm{GC}$ & Long-segment \\
\hline HSCR 75 & Sporadic & 6 & $\mathrm{c} 1118 \mathrm{C}>\mathrm{T}$ & A373V & Father & Yes & GC/GA & Long-segment \\
\hline HSCR 9 & Familial & 6 & IVS $6+2 \mathrm{~T}>\mathrm{A}$ & & Mother & No & & Long-segment,TCA \\
\hline \multirow[t]{2}{*}{ HSCR 74} & Sporadic & 10 & $\mathrm{c} 1776-7 \mathrm{GG}>\mathrm{AT}$ & G592G & Father & No & $\mathrm{AC} / \mathrm{AC}$ & TCA \\
\hline & & & & G593X & Father & No & & \\
\hline HSCR 27 & Sporadic & 12 & c2220insC & 740insC & de novo & No & GC/GA & TCA \\
\hline HSCR 39 & Sporadic & 12 & c2266insT & 756ins T & de novo & No & GC/GA & Long-segment \\
\hline HSCR 3 & Familial & 13 & $\mathrm{c} 2371 \mathrm{~T}>\mathrm{A}$ & Y791N & Mother & Yes & & Not available \\
\hline HSCR 8 & Sporadic & 13 & IVS13 + 19T $>C$ & & Mother & Yes & $\mathrm{AC} / \mathrm{GA}$ & Short-segment \\
\hline HSCR 70 & Sporadic & 19 & $\mathrm{c} 3185 \mathrm{~A}>\mathrm{G}$ & Y1062C & Father & Yes & $\mathrm{AC} / \mathrm{GC}$ & Short-segment \\
\hline
\end{tabular}

${ }^{a}$ The term "familial" is applied when more than one affected member with Hirschsprung disease is present in the same family. Otherwise, when there is no family history, the cases are considered as sporadic.

$b$ "Presence in other family members" refers to additional family members besides the parent from who the patient inherits the mutation.

to those previously published were obtained for the group of patients without RET mutation, supporting again the association of the AC haplotype with HSCR. However, interestingly no distortion of the transmission of alleles from parents to affected offspring was found in the group of triads with a RET mutation, which would support a major role for such mutations in the pathogenesis of the disease.

\section{DISCUSSION}

Several studies in different populations have identified a variety of mutations in different domains of the RET proto- oncogene associated with HSCR. It was demonstrated that the molecular mechanism of RET dysfunction highly depends on the location of the mutation. While HSCR mutations detected in the extracellular domain of RET affected the protein folding, maturation and intracellular transport to the plasma membrane, mutations in the tyrosine kinase domain resulted in impairment of its kinase activity or interference with its binding to cytosolic proteins which would be critical to signaling events. ${ }^{26}$ This is the first study in which a complete RET mutational screening is reported in a series of consecutive HSCR cases coming from Spain. We have identified 10 germline mutations among 106 patients, resulting in a mutation frequency 
A. Family HSCR 3

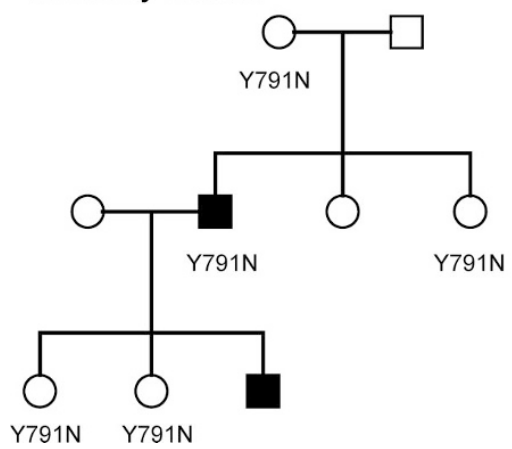

B. Family HSCR 9

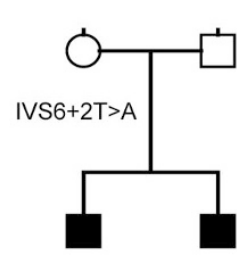

IVS6+2T $>A \quad$ IVS6+2T $>A$
C. Family HSCR 128

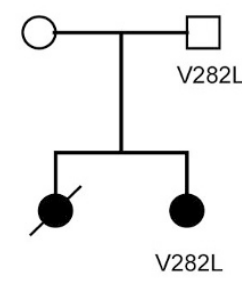

Fig. 3. Pedigrees of familial HSCR cases with mutations in the RET proto-oncogene.

Table 3

Distribution of RET transmitted and nontransmitted haplotypes in the groups of HSCR patients with and without traditionl RET mutations

\begin{tabular}{cccccc}
\hline & $\begin{array}{c}\text { HSCR patients withouttraditional } \\
\text { RET mutations }\end{array}$ & & \multicolumn{2}{c}{$\begin{array}{c}\text { HSCR patients withtraditional } \\
\text { RET mutations }\end{array}$} \\
\cline { 2 - 3 } & $\begin{array}{c}\text { Transmitted } \\
\text { alleles }\end{array}$ & $\begin{array}{c}\text { Nontransmitted } \\
\text { alleles }\end{array}$ & & $\begin{array}{c}\text { Transmitted } \\
\text { alleles }\end{array}$ & $\begin{array}{c}\text { Nontransmitted } \\
\text { alleles }\end{array}$ \\
\hline AC & 105 & 32 & & 7 & 3 \\
GC & 36 & 64 & & 4 & 8 \\
GA & 27 & 61 & & 3 & 3 \\
& $\chi^{2}=59.57, P<10^{-9}$ & & $\chi^{2}=2.93, P=0.23$ \\
\hline
\end{tabular}

Haplotypes were constituted by the combination of the two variants located within the promoter region of RET $(-200 \mathrm{~A}>\mathrm{G}$ and $-196 \mathrm{C}>\mathrm{A})$.

of $10 \%$ in sporadic patients and $23 \%$ in familial cases. Our frequencies are therefore concordant with previous studies that report values of up to $50 \%$ in familial forms and $3-35 \%$ in sporadic cases. ${ }^{2,9,10,12,22}$

We have found three missense mutations affecting the extracellular domain of RET, V145L, V282L and A373V, which might act affecting the protein folding, maturation or intracellular transport to the plasma membrane, as it has been previously proposed. Of note, V145 is one of the high conserved residues in the consensus sequence of the cadherin-like domain 1 (CLD1), having a structural role in one of the hydrophobic cores present in the extracellular region of the protein. ${ }^{27}$ The rest of missense mutations are located in the intracellular tyrosine kinase domain, being especially interesting a replacement of tyrosine in position 1062 for cysteine. This mutation had been previously described by $\mathrm{Wu}$ et al. in identical twin brothers. ${ }^{25}$ Tyrosine 1062 represents an autophosphorylation site of the RET protein and is a major binding site for the Shc adaptor proteins that are involved in the signaling pathways triggered by RET. ${ }^{28}$ Consequently, several downstream pathways would be disturbed with the presence of the conservative mutation Y1062C.

Also worthy of note is the finding of a double sequence variation in exon 10 (c.1776-7GG>AT) which generates two different replacements, the silent G592G and the nonsense G593X. Such nonsense mutation would result in the protein truncation at the transmembrane and tyrosine kinase domains, which may cause the loss of protein expression in the plasma membrane.

Finally two intronic mutations were also localized, IVS6 + $2 \mathrm{~T}>\mathrm{A}$ and IVS13 $+19 \mathrm{~T}>\mathrm{C}$. The first mutation in intron 6 involves the second base following the exon, abolishing the canonical "gt" donor site necessary for correct splicing. On the other hand, in silico studies predicted that mutation in intron 13 also abolishes a donor splice site. Therefore, we have arguments enough to postulate that both are splice site mutations capable of causing aberrant splicing.

Analysis of parental DNA in sporadic cases showed that all these mutations previously mentioned are inherited from either the unaffected mother or father, and also appeared in apparently healthy siblings. Therefore, expression and penetrance of the disease in RET mutation carriers were variable within HSCR pedigrees, according to a complex model of inheritance. In contrast, the two insertions are both de novo mutations, thus suggesting that they could be directly related with the disease phenotype. The insertions occurred in exon 12 and are expected to abolish tyrosine kinase activity.

It is also interesting to point out that, at least $8 / 12$ patients with RET mutations (67\%) presented long-segment/TCA phenotype. This finding is in accordance with those previously reported where, mutations in RET are associated with longsegment phenotype more frequently than the short-segment phenotype. ${ }^{8-13}$

Because of the recent discovery of a predisposing haplotype for HSCR located at the 5'region of RET we have also proceeded to analyze the distribution in carriers and noncarriers of germline RET mutations. In a previous report we had shown an association of the combination $-200 \mathrm{~A} /-196 \mathrm{C}$ to the sporadic forms of HSCR in our series. ${ }^{19}$ This association was supported not only by the results from comparative studies between our HSCR patients and normal controls, but also from the TDT analysis in the HSCR triads. Moreover, luciferase expression assays revealed a significantly depressed activity for the HSCR-linked haplotype at $-200 /-196$ in comparison with other combinations associated with controls, which might be indicative of functional mechanism for both promoter variants. Our present results support the previous find- 
ings since a significant over-transmission of the risk haplotype was detected when comparing transmitted versus nontransmitted alleles in the group of HSCR triads without mutation. However, no distortion of the transmission of alleles from parents to affected offspring was found in the group of mutated families. Moreover, of note, we have observed that the two patients with de novo mutations, did not present the risk haplotype, suggesting that the mutation alone could play a causative role in the development of the disease phenotype in those patients. By contrast, except for one patient, the remaining sporadic cases presented the RET mutations in trans with the risk haplotype.

On the other hand, the finding of association to HSCR across the RET intron 1 leaded to search for functional elements in such region. ${ }^{29}$ Emison et al. showed that a common noncoding RET variant ( $\mathrm{rs} 2435357$, IVS1 $+9277 \mathrm{~T}>\mathrm{C}$ ) within a conserved enhancer-like sequence in intron 1 was significantly associated with HSCR susceptibility. They also found that the T-allele reduced in vitro enhancer activity markedly, decreasing RET transcription. Of note, such variant had been also found to be associated to HSCR in our series. ${ }^{19}$ Moreover, we had observed that among the 13 different haplotypes generated in our previous study, the allele IVS1 + 9277T was exclusively present in the "HSCR haplotype" and in complete linkage disequilibrium with the $-200 \mathrm{~A} /-196 \mathrm{C}$ promoter combination. Therefore, all these data would be concordant with a complex additive model of inheritance in which the confluence of several factors, such as RET coding mutations or specific haplotypes, are necessary to produce the phenotype. In these terms, we could justify the presence of RET coding mutations in healthy members of the HSCR families: such lowpenetrance mutations would be necessary but not sufficient to produce the phenotype, and the additional presence of the "HSCR haplotype" could contribute to the manifestation of the disease. Moreover, mutational events occurring in other candidate genes still unidentified, such those involved in the development of enteric nervous system, may have also a critical role.

\section{ACKNOWLEDGMENTS}

We would like to thank the families who participated in the study. This study was partially funded by grants PI040266 and PI051291 from the Fondo de Investigación Sanitaria, Spain.

\section{References}

1. Badner JA, Sieber WK, Garver KL, Chakravarti A. A genetic study of Hirschsprung disease. Am J Hum Genet 1990;46:568-580.

2. Chakravarti A, Lyonnet S. Hirschsprung Disease. In: Scriver CR, Beaudet AL, Sly WS, Valle D, et al. The metabolic and molecular bases of inherited disease. New York: McGraw-Hill, 2002:6231-6255.

3. Wakamatsu N, Yamada Y, Yamada K, Ono T, et al. Mutations in SIP1, encoding Smad interacting protein-1, cause a form of Hirschsprung disease. Nat Genet 2001; 27:369-370.

4. Amiel J, Laudier B, Attie-Bitach T, Trang H, et al. Polyalanine expansion and frameshift mutations of the paired-like homeobox gene PHOX2B in congenital central hypoventilation syndrome. Nat Genet 2003;33:459-461.
5. Brooks AS, Bertoli-Avella AM, Burzynski GM, Breedveld GJ, et al. Homozygous Nonsense Mutations in KIAA1279 Are Associated with Malformations of the Central and Enteric Nervous Systems. Am J Hum Genet 2005;77:120-126.

6. Ceccherini I, Bocciardi R, Luo Y, Pasini B, et al. Exon structure and flanking intronic sequences of the human RET proto-oncogene. Biochem Biophys Res Commun 1993; 196:1288-1295.

7. Takahashi M. The GDNF/RET signaling pathway and human diseases. Cytokine Growth Factor Rev 2001;12:361-373.

8. Yin L, Barone V, Seri M, Bolino A, et al. Heterogeneity and low detection rate of RET mutations in Hirschsprung disease. Eur J Hum Genet 1994;2:272-280.

9. Attie T, Pelet A, Edery P, Eng C, et al. Diversity of RET proto-oncogene mutations in familial and sporadic Hirschsprung disease. Hum Mol Genet 1995;4: 1381-1386.

10. Angrist M, Bolk S, Thiel B, Puffenberger EG, et al. Mutation analysis of the RET receptor tyrosine kinase in Hirschsprung disease. Hum Mol Genet 1995;4:821-830.

11. Seri M, Yin L, Barone V, Bolino A, et al. Frequency of RET mutations in long- and short-segment Hirschsprung disease. Hum Mutat 1997;9:243-249.

12. Svensson PJ, Molander ML, Eng C, Anvret M, et al. Low frequency of RET mutations in Hirschsprung disease in Sweden. Clin Genet 1998;54:39-44.

13. Fitze G, Cramer J, Ziegler A, Schierz M, et al. Association between c135G/A genotype and RET proto-oncogene germline mutations and phenotype of Hirschsprung's disease. Lancet 2002;359:1200-1205.

14. Borrego S, Saez ME, Ruiz A, Gimm O, et al. Specific polymorphisms in the RET proto-oncogene are over-represented in patients with Hirschsprung disease and may represent loci modifying phenotypic expression. J Med Genet 1999;36:771-774.

15. Borrego S, Ruiz A, Saez ME, Gimm O, et al. RET genotypes comprising specific haplotypes of polymorphic variants predispose to isolated Hirschsprung disease. J Med Genet 2000;37:572-578.

16. Borrego S, Wright FA, Fernandez RM, Williams N, et al. A founding locus within the RET proto-oncogene may account for a large proportion of apparently sporadic Hirschsprung disease and a subset of cases of sporadic medullary thyroid carcinoma. Am J Hum Genet 2003;72:88-100.

17. Fitze G, Appelt H, Konig IR, Gorgens H, et al. Functional haplotypes of the RET proto-oncogene promoter are associated with Hirschsprung disease (HSCR). Hum Mol Genet 2003;12:3207-3214.

18. Burzynski GM, Nolte IM, Bronda A, Bos KK, et al. Identifying Candidate Hirschsprung Disease-Associated RET Variants. Am J Hum Genet 2005;76:850-858.

19. Fernandez RM, Boru G, Pecina A, Jones K, et al. Ancestral RET haplotype associated with Hirschsprung's disease shows linkage disequilibrium breakpoint at -1249 . J Med Genet 2005;42:322-327.

20. Garcia-Barcelo M, Ganster RW, Lui VC, Leon TY, et al. TTF-1 and RET promoter SNPs: regulation of RET transcription in Hirschsprung's disease. Hum Mol Genet 2005;14:191-204.

21. Griseri P, Bachetti T, Puppo F, Lantieri F, et al. A common haplotype at the 5 ' end of the RET proto-oncogene, overrepresented in Hirschsprung patients, is associated with reduced gene expression. Hum Mutat 2005;25:189-195.

22. Pelet A, de Pontual L, Clement-Ziza M, Salomon R, et al. Homozygosity for a frequent and weakly penetrant predisposing allele at the RET locus in sporadic Hirschsprung disease. J Med Genet 2005;42:18.

23. Dracapoli NH, Haines JL, Korf BR, Moir DT, et al. Current Protocols in Human Genetics. New York: Wiley \& Sons, 1994.

24. Fitze G, Schierz M, Kuhlisch E, Schreiber M, et al. Novel intronic polymorphisms in the RET proto-oncogene and their association with Hirschsprung disease. Hum Mutat 2003;22:177.

25. Wu TT, Tsai TW, Chu CT, Lee ZF, et al. Low RET mutation frequency and polymorphism analysis of the RET and EDNRB genes in patients with Hirschsprung disease in Taiwan. Hum Genet 2005;50:168-174.

26. Pelet A, Geneste O, Edery P, Pasini A, et al. Various mechanisms cause RET-mediated signaling defects in Hirschsprung's disease. J Clin Invest 1998;101:1415-1423.

27. Anders J, Kjar S, Ibanez CF. Molecular modeling of the extracellular domain of the RET receptor tyrosine kinase reveals multiple cadherin-like domains and a calciumbinding site. J Biol Chem 2001;276:35808-35817.

28. Asai N, Murakami H, Iwashita T, Takahashi M, et al. A mutation at tyrosine 1062 in MEN2A-Ret and MEN2B-Ret impairs their transforming activity and association with shc adaptor proteins. J Biol Chem 1996;271:17644-17649.

29. Emison ES, McCallion AS, Kashuk CS, Bush RT, et al. A common sex-dependent mutation in a RET enhancer underlies Hirschsprung disease risk. Nature 2005;434: $857-863$. 